\title{
Réflexions sur les vortex de vidange
}

\section{Remarks on drain vortex phenomena}

\author{
par F. BIESEL \\ INGÉNIEUR AU LABORATOIRE DAUPhiNOIS D'hydRAULIQUe (GRENOBLE)
}

\begin{abstract}
Le caractère explosif et la ténacité des vortex de vidange. - Les contradictions thériques qu'évoque ce phénomène. - L'intérêt de la notion de concentration du moment cinétique. - Etude théorique, dans divers cas, de l'évoIntion d'une circulation primitivement diffuse dans le fluide. - Justification de l'emballement du phénomène. - Difficultés is expliquer la naissance du vortex et sa ténacité.
\end{abstract}

\begin{abstract}
The explosive character and tenacity of dratn vortices. Theoretical contradictions that arise from this phenomenon. The importance of the idea of the concentration of the moment of momentum. The theoretical studly in different cases of a circulation that is originally dispersed in the flow. A justification of the sudden violence of the phenomenon. Difficulties in explaining how it arises and its tenacify.
\end{abstract}

Le vortex de vidange est certainement l'un des phénomènes les plus familiers de l'ingénieur hydraulicien, qui peut souvent l'observer lorsqu'à un écoulement à surface libre se superpose localement un écoulement dans le sens vertical, contròlé par un orifice relativement étroit, disposé à une certaine profondeur. La surface libre forme une sorte d'entonnoir plus ou moins nettement creusé, el animé d'une vitesse de rotation eroissant rapidement lorsque l'on se rapproche du centre. Si l'on dispose $\dot{a}$ la surface du courant, assez loin à l'amont, un corps flottant, on le voit d'abord se déplacer assez lentement en direction de la zone lourbillonnaire, puis, petit à petit, il est pris dans la ronde, tourne de plus en plus vite au fur ef à mesure qu'il se rapproche du centre et, brusquement, il se trouve véritablement aspiré vers le fond.

Le phénomène n'est pas toujours absolument permanent: le vortex apparaît, s'amplifie, puis il semble diminuer, disparaît presque pour se reformer et ainsi de suite. L'expérience montre, en tout cas, que si c'est là un phénomène éminemment capricieux, c'est aussi un phénomène particulièrement tenace auquel il est très difficile de s'opposer.

Les études faites sur ce sujet sont évidemment assez nombreuses, et nous citerons seulement pour mémoire, d'une part les chapitres qu'y a consacrés M. Boussse dans son cours, d'autre part le vortex combiné de Rankine qui donne une solution simple cn fluide parfait.

Cependant, dans tout ce que nous avons pu voir jusqu'ici, nous n'avons pas trouve de caleul quantitalif précis sur l'hydrodynamique des vortex à proprement parler. Le phénomène de la concentration du moment cinétique était bien connu et signalé, mais il est rare, semble$t$-il, que les auteurs aient été jusqu'à donner des formules simples permettant des applications numériques à des cas concrets.

Quelle est l'origine du phénomène? Peul-on expliquer le caractère parfois explosif de son apparition? Existe-L-il des moyens de l'éviter?

Nous ne prétendons pas apporter à toutes ces questions une réponse satisfaisante. Notre but est seulement de faire part des réflexions et des remarques que nous ont suggérées nos études dans ce domaine, et de susciter peut-être les expériences qui pourraient confirmer ou infirmer certaines de nos conclusions, facilitant ainsi la poursuite de recherches plus appro. fondies.

Nous nous sommes attachés, dans la mesure du possible, à envisager le problème sous son aspect pratique et à aboutir à des résultats utilisables dans l'industrie, par exemple en ce qui concerne les moyens de s'opposer à l'apparition de vortex de vidange. Une telle préoccupation 
est trop souvent négligée dans ce domaine si l'on en excepte certains travaux sur l'intérêt qu'il peut $y$ avoir parfois à entretenir des vortex de vidange.

C'est un peu dans le même esprit que nous avons expurgé notre exposé des développements mathématiques sur lesquels nous avons étayé nos remarques, mais dont le détail n'est pas indispensable à la compréhension de la théorie. Les lecteurs que cette question intéresse trouveront rassemblées en annexe ces justifications mathématiques.

\section{I. - GÉNÉRALITÉS}

Au seuil des quelques remarques théoriques que nous allons présenter ci-après, il convient de situer le probleme par rapport aux lois de l'hydrodynamique classique.

On sait d'abord que la création de circulation est un phénomène anormal dans l'écoulement des fluides parfaits. La formation d'un vortex de vidange, malgré de multiples précautions pour assurer une alimentation bien symétrique, son amplification le portant à des intensités parfois considérables, semblent donc échapper quelque peu aux moyens d'analyse habituels. Cependant, le théorème classique de la conservation du moment cinétique paraît constituer une mine particulièrement abondante d'explications.

Supposons, en effet, qu'autour d'un puits, un anneau liquide soit déjà animé d'un certain mouvement de rotation; lorsque, sucé par le puits, son diamètre diminuera, l'application du théorème montre que sa vitesse de rotation augmentera corrélativement. C'est ce que nous appellerons la concentration du moment cinctique, phénomène qui permet d'expliquer qu'à partir du moment ou existe, pour une raison ou pour une autre, une rotation même à peine perceptible très loin du puits, on puisse avoir au voisinage du centre une rotation très rapide.

\section{1. - CAS DES ECOULEMENTS DE REVOLUTION}

La concentration du moment cinétique expliquerait peut-être, par exemple, que des vitesses de rotation aussi faibles que celles de la terre puissent suffire à amorcer des vortex très rapides. On aurait, en effet, observé que, lorsque les conditions d'alimentation sont très symétriques, les vortex ont tendance à démarrer d'un côté dans l'hémisphère nord, et de l'autre dans l'hémisphère sud. Nos premiers calculs ont eu pour but de contrôler la vraisemblance ou l'impossibilité d'une telle influence.

\section{A) Hypothèse di FLUIDF PARFAit}

\section{a) Problème plan.}

Pour simplifier, nous négligerons d'abord la viscosité et nos calculs seront conduits comme si nous nous trouvions en fluide parfait. De plus, et toujours pour simplifier les calculs, les deux premiers cas envisagés ci-dessous se rapporteront à des problèmes plans, autrement dit à des problèmes dans lesquels la profondeur du bassin est supposée constante, le puits se présentant sous la forme d'un petit tube vertical, par exemple une crépine de pompe d'une hauteur égale à la profondeur de la nappe d'eau où elle plonge.

Nous avons alors étudié, dans deux cas dis- tincts, l'évolution d'une circulation primitivement diffuse dans le fluide.

Si la rotation initiale obéit à la loi du vortex, en d'autres termes si les vitesses tangentielles sont inversement proportionnelles au rayon, il ressort du calcul que, dans son ensemble, le mouvement est permanent. En un point donné la vitesse est constante. Par contre, si l'on considère un anneau de fluide déterminé, sa vitesse de rotation augmente évidemment au fur et à mesure qu'il approche du puits.

Admettons maintenant que la rotation initiale se fasse en masse. Ce cas correspond, par exemple, à une expérience faite à un des pôles terrestres, la rotation en masse étant alors celle de la terre; ou encore, et moyennant une prise en compte adéquate la force du Coriolis, ce cas correspond à une expérience faite en un point quelconque, la rotation en masse fictive étant prise égale au produit de la rotation de la terre par le sinus de la latitude.

Le phénomène de concentration du moment cinétique amène cette fois une accélération du mouvement d'ensemble du fluide; à la rotation en masse initiale, très lente, se superpose une rotation du type vortex croissant proportionnellement au temps. Il en résulte une accélération très rapide, dans le temps et dans l'espace, des anneaux liquides se rapprochant de l'axe de rotation qui coïncide avec l'axe du puits. En un 
point donné les vitesses vont donc constamment en croissant; le mouvement tend à s'emballer. Pour fixer les idées, nous supposerons avoir affaire à un puits d'une puissance de $100 \mathrm{~cm}^{2}$ par seconde: ce qui signifie, par exemple, qu’à $20 \mathrm{~cm}$ de l'orifice, la vitesse centripéte de l'eau atteint $5 \mathrm{~cm} / \mathrm{sec}$. L'application de notre calcul théorique montre que, sur un cercle de $2 \mathrm{~mm}$ de diamitre, la vitesse de rotation passe en 4 secondes de celle de la terre (un tour en $85000 \mathrm{sec}$ ) à un tour/seconde environ. Ceci est considérable et bien en accord avec l'allure explosive que prisente souvent le phénomène.

\section{b) Problemes à trois dimensions.}

Les cas suivants ont été étudiés pour un écoulement à trois dimensions, ce qui correspond à l'hypothèse beaucoup plus intéressante du point de vue pratique d'un puits situé au tond d'une nappe d'eau. Là encore, nous avons recherché comment évoluait une rotation initiale ayant la loi $\mathrm{du}$ vortex. A nouveau, nous avons constalé que ce type de rotation ne s'emballait pas, mais restait constant. Puis, nous sommes passés au cas où la rotation initiale se fait en masse, ce qui nous a conduit aux conclusions suivantes résumées par la planche $I$.

Tout autour de la région centrale, intéressée
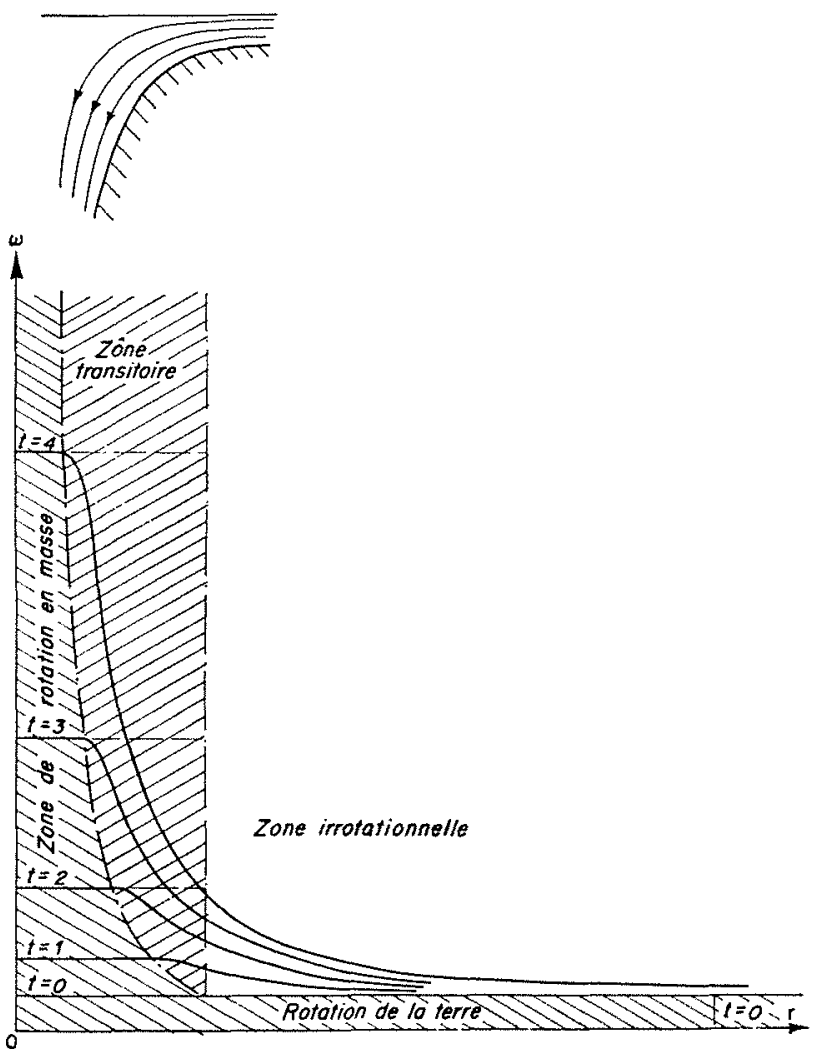

Planche I par l'influence des mouvements verticaux descendant vers le puits, on constate que la rotation en masse s'accélère et forme un noyau dont le diamètre diminue d'ailleurs constamment. A l'extérieur de cette zone la rotation s'accélère par la superposition, à la rotation en masse initiale, d'une rotation en vortex croissant linéairement a vec le temps. Dans ce dernier cas, la vitesse de rotation du noyau central tournant en masse crô̂t exponentiellement avec le temps, ce qui est bien en accord avec les démarrages parfois brulaux qui caractérisen! les vortex de vidange.

Ces études préliminaires ont montré en définitive qu'il suffisait de causes à peine pereeptibles pour provoquer au droit du puits un vortex appréciable. Par conséquent, il n'est pas nécessaire de recourir à des forces occultes, ou d'imaginer que l'énergie de la chute à travers l'orilice sert à entretenir le mouvement de rotation. Cependant, une remarque essentielle s'impose: les calculs montrent bien qu'une tres fable rolation, initialement diffuse dans la masse du fluide, suffit, en se concentrant au droit du puits, à créer un mouvement de rotation intense : ils montrent par contre aussi que, si celte rotation ne préexiste pas sous une forme diffuse ou aulre, elle ne peut pas se créer d'elle-même.

En particulier, si les nappes d'eau s'étendant à l'infini étaient dénuées de toute rotation initiale, le vortex devrait finir par s'éteindre et ne pas renaître. Les considérations qui précèdent ne permettent donc absolument pas d'expliquer par quel mécanisme un vortex une fois amorcé semble aussitôt s'emballer, et se montre d'autant plus difficile à arrêter qu'il a pris plus d'élan. Certes, les zones très éloignées de l'axe ont toujours une vitesse de rotation qui est celle de la terre, mais pourquoi alors voil-on des vortex prendre naissance et s'emballer aussi bien dans un sens que dans l'autre?

\section{3) INFLUENCE DE IA VISCOSITE:}

Nous avons repris les mêmes calculs en essayant de tenir compte de la viscosité. On pourrail supposer, en effet, que les anneaux liquides se trouvant près du puits, où ils ont une vitesse de rotalion tris grande, cèdent une partie de cette vitesse de rotation aux couches extérieures voisines, et qu'ainsi le mouvement de rotation du vortex s'entretiendrait sans faire appel à des apports extérieurs.

Les équations que nous avons établies tiennent compte de l'influence réciproque des différents anneaux liquides, mais ne tiennent pas compte des effets de paroi sur lesquels nous aurons à revenir. Dans les conditions du calcul, on constate que, qualitativement, rien n'est changé : les mouvements de rotation s'emballent 
de la même facon que lorsque la viseosité est négligeable. En particulier, si les zones éloignées du liquide ne tournent pas, le vortex devrail finir par s'éteindre de lui-même rapidement, après que les régions initialement animées de mouvement de rotation aient disparu dans le puits.

\section{III. - ECOULEMENTS NON DE REVOLUTION}

Jusçu'ici nous nous élions limités pour ces éludes théoriques aux problèmes strictement de révolution, c'est-à-dire que les bassins envisagés étaient supposés parfaitement circulaires et percés en leur centre d'un orifice axial. D'après nos premières conclusions, il semble que, pour ce type de bassin, seule la rotation de la terre soit susceptible de provoquer la formation de vortex, et qu'en particulier, toute rotation amorcée dans le sens contraire soit appelée à disparaître. Il est donc intéressant de chercher à savoir ce que deviennent ces conclusions dans le cas d'un récipient de forme quelconque. Ce dernier cas est évidemment beaucoup plus complexe et on ne peut pas le traiter sous une forme aussi rigoureuse que pour un récipient de révoIution. Cependant, ainsi que nous allons le voir, son analyse se révèle riche d'enseignements.

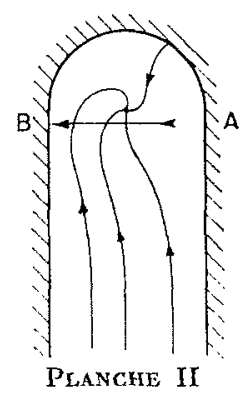

Sur la planche 2 nous avons représenté schématiquement l'écoulement dans un canal en cul de sac terminé par un demi-cercle au centre duquel se trouve un puits. Supposons qu'un vortex de sens opposé à celui de la rotation terrestre y soit amorcé et s'entretienne. En première analyse, il semble impossible que les masses d'eau veninn de l'infini par ce canal, et qui ont à l'origine un moment cinélique dans un certain sens par rapport à l'axe vertical passant par le puits, puissent toul à coup se trouver en avoir un de sens contraire lorsqu'elles s'engagent dans le vortex. En effet, on pourrait s'attendre à ce que, les masses d'eau toumant autour du puits une fois absorbées, l'alimentation en cau dirigée axialement tende à ramener la symétrie, ou une rotation de sens inverse, dans l'écoulement. Comment s'explique donc ce paradoxe apparent de la non-conservation du moment cinćtique pour les masses d'eau? Il est facile de répondre à cette question.

Dans la région A de l'éconlement, les filets d'eau ralentissent par suite de la rencontre du courant incident avec le courant du vortex. Dans la région $\mathrm{B}$, le courant est au contraire accéléré; la pression en A est donc plus grande qu'en B et la résultante de la réaction des parois sur le fluide est une force qui, dirigée de $A$ vers $B$, communique à la veine liquide un moment de rotation ainsi que le montre le classique théorème des quantités du moment cinétique. Pour un fluide parfait, cette réaction est juste celle qu'il faut pour entretenir le vortex, c'est-à-dire donner aux masses d'eau incidentes le « coup de pouce » nécessaire pour qu'elles se mettent à tourner sur les traces de leurs devancières et à la mème vitesse. Cette remarque, très banale en soi, est très utile pour expliquer le phénomène de l'entretien du vortex de vidange. Elle montre que, même en fluide parfait, le vortex une fois créé peut subsister. Nous avons donc presque notre réponse à la question que nous avons posće plus haut. Mais cetle réponse n'est pas encore entièrement salisfaisante car le vortex ne se contente pas de subsister lorsqu'il est créé, mais va même jusqu'à prendre naissance à partir de perturbations initiales infimes et à alteindre une ritesse de rotation importante.

Il est difficile de donner avec certilude une explication à ce dernier phénomène, qui d'aillcurs gagnerail à être précisé, voire même confirmé, sur des installations d'essai présentant rigoureusement toutes garanties de symétrie. Nous proposons cependant la suivante : en fluide parfait, la différence de pression entre $A$ et $B$ est juste celle qu'il faut pour donner au fluide revenant de l'amont la déviation nécessaire. Mais, lorsque le fuide est réel, les vitesses d'écoulement sur le fond et dans la masse sont moindres que les vilesses en surface; ainsi la diffèrence de pression (ou de niveau si l'on est en surface libre) est trop importante pour les parties profondes du courant. Ces parlies profondes sont en effet soumises sensiblement à la même force de déviation que les parties superficielles, mais conme elles vont beaucoup moins vite, elles dévient plus; car, en quelque sorte, chaque particule subit plus longtemps le gradient de pression déviateur. Ainsi pourrait-on comprendre que la différence de niveau, qui est juste suffisante pour entretenir le mouvement dans le cas d'un fluide parfait, soit excessive dans le cas d'un fluide réel et conduise à un emballement du phénomène. 
Aux questions que nous avons posées au début de cet exposé, nous n'avons certes pas apporté de réponse parfaitement satisfaisante. Certains aspects des problèmes soulevés apparaissent peut-être plus clairement, d'autres demeurent encore bien obscurs. C'est ainsi que, d'une facon générale, l'étude théorique révèle fidèlement, et explique dans une large mesure, le caractère ex- plosif du phénomène qui, une fois amorcé, s'emballe à un rythme apparemment irrésistible. Par contre, la naissance et la ténacité du phénomène tiennent à des causes qui, si elles sont parfois soupconnées, ont encore largement de quoi exciter la curiosité et la perspicacilé des chercheurs.

\section{$A N N E X E$}

\section{ETUDES THÉORIQUES SCHÉMATIQUES DE QUELQUES CAS TYPES}

Dans ce qui suit nous nous limitons à l'étude des écoulements présentant une symétrie de rotation par rapport à un axe vertical $\mathrm{OZ}$.

Nous ferons une hypothèse complémentaire consistant à admettre que l'écoulement se fait par tranches cylindriques verticales : un cylindre liquide, ayant pour axe $\mathrm{OZ}$, se déformera au cours du mouvement sans cesser d'être un cylindre d'axe $O Z$. On admet en d'autres termes que les vitesses radiales ne dépendent pas de la profondeur.

On connaît au moins quatre types d'écoulements potentiels de révolution pour lesquels cette condition est rigoureusement salisfaite. Ce sont :

1. - L'écoulement plan autour d'un puits (axial).

Dans cel écoulement on a :

$$
\mathrm{V}_{r}=-\frac{\mathrm{K}}{r} \quad \mathrm{~V}_{t}=0 \quad \mathrm{~V}_{z}=0
$$

avec :

$V_{r}=$ composante radiale de la vitesse,

$V_{t}=$ composante taugentielle de la vitesse,

$\mathrm{V}_{z}=$ composante selon l'axe $\mathrm{OZ}$ de la vilesse.

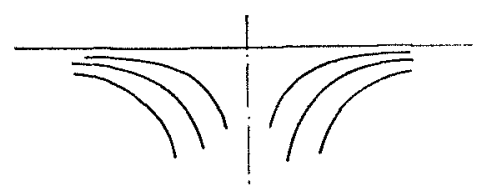

Fig. 1

2. - L'analogue, pour les écoulements de révolution, de l'écoulement dans un angle pour les écoulements plans (voir fig. 1) : on a alors :

$$
\mathrm{V}_{r}=-\mathrm{Cr} \quad \mathrm{V}_{t}=0 \quad \mathrm{~V}_{z}=2 \mathrm{C} z
$$

3. - Le vortex irrotationnel autour d'un axe pour lequel :

$$
\mathrm{V}_{r}=0 \quad \mathrm{~V}_{t}=\frac{\mathrm{K}}{r} \quad \mathrm{~V}_{z}=0
$$

4. - A noter enfin parmi les mouvements rotationnels, mais dynamiquement possibles, toutes les rotations pures de la forme :

$$
\mathrm{V}_{r}=0 \quad \mathrm{~V}_{t}=f(r) \quad \mathrm{V}_{z}=0
$$

On voit que notre liypothèse est rigoureusement satisfaite pour un certain nombre de mouvements dynamiquement possibles el pour toutes leurs combinaisons par addition.

En fait, dans le cas général où les profondeurs seront une fonction queleonque de $r$, cette hypothise ne sera plus rigoureusement vraic; il est à noter cependant que, pour des formes de fond correspondant bien au genre de problène étudie (comme celles représentées sur la figure 2), on retrouve un écoulement du type (1) ci-dessus dans la région $A$, et un écoulement du type (2) dans la région $B$, tout ceci combiné, par exemple, avee des rotations des types (3) ou (4).

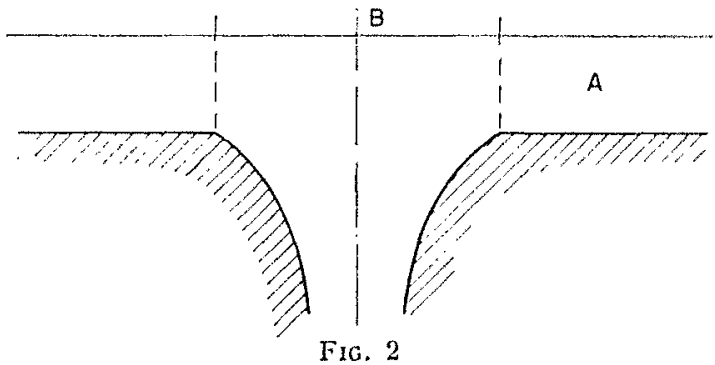

En définitive l'hypothèse du mouvement par tranches cylindriques verticales, quoique certainement fausse en toute rigueur, doit conduire à des résultats en assez bon accord avec la réalité. Notons encore, à propos de cette hypothèse, 
qu'elle implique que l'on néglige complètement les frottements sur le fond (quoique l'on ne néglige pas nécessairement la viscosité du fluide).

En harmonie avec cette dernière remarque, nous supposerons également que les vitesses tangentielles ne dépendent pas de la profondeur.

Une fois ces hypothèses admises, nous allons étudier les lois de l'hydrodynamique des écoulements considérés. Pour cela, il nous suffira d'écrire, pour chaque anneau ou cylindre de liquide, l'équation de la conservation du moment cinétique relatif à l'axe $\mathrm{OZ}$.

Examinons donc l'évolution d'un anneau de masse $\delta^{2} m$ ayant une épaisseur o $r$, une hauteur $\delta h$,et un rayon moyen $r$ au temps $t$.

Son moment cinétique autour de $\mathrm{OZ}$ est :

$$
\hat{\delta}: \mathfrak{M}=r \stackrel{\circ}{\circ} m \mathrm{~V}_{t}
$$

Le couple visqueux agissant sur l'anneau est :

$$
\begin{aligned}
& \delta^{2} \mathrm{M}=\frac{\partial}{\partial r}\left[\mu\left(\frac{\partial \mathrm{V}_{t}}{\partial r}-\frac{\mathrm{V}_{t}}{r}\right) \times 2 \pi r^{2}\right] \delta r \delta h \\
& =2 \pi \mu r^{2}\left[\frac{\partial^{2} \mathrm{~V}_{t}}{\partial r^{2}}+\frac{1}{r} \frac{\partial \mathrm{V}_{t}}{\partial r}-\frac{1}{r^{2}} \mathrm{~V}_{t}\right] \delta r \delta h
\end{aligned}
$$

Ecrivons que la vitesse de variation de $\delta^{2} \mathfrak{K}$ est égale à $\delta^{2} \mathrm{M}$ :

$\frac{\mathrm{D}}{\mathrm{D}_{t}}\left(r \partial^{2} m \mathrm{~V}_{t}\right)=2 \pi \mu r^{2}\left[\frac{\partial^{2} \mathrm{~V}_{t}}{\partial r^{2}}+\frac{1}{r} \frac{\partial V_{t}}{\partial r}-\frac{1}{r^{2}} V_{t}\right]$

Cette relation devient après développement (et compte tenu de la relation $\left.\delta^{2} m=2 \pi p r \delta r \delta h\right)$ :

$$
\begin{aligned}
\frac{\partial V_{l}}{\partial t}=\nu\left(\frac{\partial^{2} V_{t}}{\partial r^{2}}+\right. & \left.\frac{1}{r} \frac{\partial V_{l}}{\partial r}-\frac{1}{r^{2}} V_{t}\right) \\
& -V_{r}\left(\frac{\partial V_{t}}{\partial r}+\frac{1}{r} V_{t}\right)
\end{aligned}
$$

Cette formule va nous permettre de traiter rapidement quelques cas particuliers :

\section{A) VISCOSITÉ NÉGLIGÉI:}

$$
\text { 1. }-\mathrm{V}_{r}=-\frac{\mathrm{K}}{r} \text {. C'est le problème plan }
$$

d'un puits de puissance $K$ situé au centre de coordonnées. L'équation (1) devient :

$$
\frac{\partial V_{t}}{\partial t}=\mathrm{K}\left(\frac{1}{r} \frac{\partial V_{t}}{\partial r}+\frac{1}{r^{2}} V_{t}\right)
$$

a) Supposons que le mouvement de rotation initial ait la loi du vortex $\mathrm{V}_{t}=(\mathrm{A} / \mathrm{r})$; il vient :

$$
\frac{\partial V_{t}}{\partial t}=0 \text {. }
$$

Le mouvement est donc permanent (c'est tout simplement le «puits-vortex» dont la possibilité est bien connue).

En conséquence, dans ce cas, en écoulement plan, en fluide parfait autour d'un puits, la vitesse de rotation pour un rayon donné reste constante. Cependant la vitesse de rotation d'un cylindre élémentaire, considéré au cours de son évolution, va en croissant, et l'augmentation de cette vitesse compense la diminution du rayon du cylindre, le moment cinćtique demeurant constant. Mais le mouvement d'ensemble est permanent : en effet, l'augmentation de la vitesse de rotation a lieu de telle façon que, lorsqu'un cylindre arrive à une certaine distance du centre, il a pris juste la vitesse qu'avaien! en ce point les anneaux qui l'y avaient précédé;

b) Supposons que le mouvement an temps $t=0$ soit un mouvement de rotation en masse. C'est, par exemple, ce qui se passerait si le bassin étudié était situé ̀̀ un pôle de la terre et bien entendu solidaire de la rotation de celle-ci. Soit : $V_{(t=0)}=\omega r$ les rotations initiales, La solution correspondante se trouve aisément, elle s'écrit :

$$
\mathrm{V}_{t}=\omega r+\frac{2 \mathrm{~K} \omega t}{r}
$$

Le mouvement n'est plus permanent; à la rotation initiale $\omega r$ se superpose un rortex irrotationnel dont la puissance croît linéairement avec le temps.

La concentration des moments a ici pour effet d'augmenter, non seulement la vitesse de rotation des cylindres liquides considérés individuellement, mais aussi celle du mouvement tourbilIonnaire pris dans son ensemble. Un exemple numérique permettra de mieux juger de l'aspect pratique du phénomène que nous venons de décrire.

Supposons que la puissance $K$ de la source soit $100 \mathrm{~cm}^{2} / \mathrm{s}$, c'est-à-dire qu'à $20 \mathrm{~cm}$ de l'origine la vitesse radiale soit de $5 \mathrm{~cm} / \mathrm{s}$, à $10 \mathrm{~cm}$ de $10 \mathrm{~cm} / \mathrm{s}$, à $5 \mathrm{~cm}$ de $20 \mathrm{~cm} / \mathrm{s}$, etc. (Nous avons en vue une expérience à une échelle modeste par exemple.)

La vitesse angulaire $\Omega$ en fonction du rayon et du temps est donnée par :

$$
\Omega=\omega\left(1+\frac{2 K t}{r}\right)=\omega\left(1+200 \frac{t}{r}\right)
$$

Cette vitesse angulaire sera, sur un cercle de $2 \mathrm{~mm}$ de diamètre $(r=0,1 \mathrm{~cm})$ :

$$
\Omega_{0.1}=(1+20.000 t)
$$

Au bout d'une seconde, la vitesse de rotation 
sur ce cercle sera donc 20.000 fois plus grande que la vitesse initiale. Au bout de 4 secondes, elle sera 80.000 fois plus grande, c'est-à-dire que si la vitesse initiale était celle de la terre (1 tour en 85.000 secondes), la vitesse sur ce cercle de $2 \mathrm{~mm}$ de rayon serait devenue, au bout de 4 secondes seulement, de l'ordre d'un tour par seconde.

Notons, par contre, que sur un cercle de $40 \mathrm{~cm}$ de diamètre par exemple, la vitesse de rolation n'est multipliée que dans le rapport de 1 ì 3 pendant ce même laps de temps de 4 secondes, et que le moment cinétique à l'intérieur du même cercle a été multiplié par 17 pendant ces 4 premières secondes.

Les conclusions de ce premier calcul sont très importantes; on peut les énoncer ainsi :

Il suffit d'une très faible «vorticité 》 diffuse sur l'ensemble du plan d'eau pour provoquer autour du puits un emballement extrèmement rapide et brutal de la rotation;

L'effet de la rotation terrestre (aussi lente soitclle) suffit pour amorcer rapidement, dans des conditions usuelles, des rotations très rapides. Il est donc probablement vrai qu'en l'absence de toute autre cause de dissymétrie, les vortex de vidange démarrent dans un sens ou dans l'autre suivant qu'ils sont dans l'hémisphère Nord ou dans l'hémisphère Sud. Il faut noter à ce sujet que les rotations ainsi induites par la rotation de la terre sont proportionnelles au sinus de la latitude.

$$
2 .-\mathrm{V}=-\mathrm{Cr}
$$

Pour mémoire :

$$
\left.\mathrm{V}_{z}=2 \mathrm{C} z \quad \text { (fig. } 1\right)
$$

L'équation (1) devient:

$$
\frac{\partial \mathrm{V}_{t}}{\partial t}=\mathrm{C}\left(\mathrm{V}_{t}+r \frac{\partial \mathrm{V}_{t}}{\partial r^{r}}\right)
$$

a) Supposons que la relation initiale soit du type «vortex»: $\mathrm{V}_{t}=(\mathrm{A} / \mathrm{r})$. Comme précédemment, nous trouvons :

$$
\frac{\partial V_{t}}{\partial t}=0
$$

L'écoulement est donc permanent et le vortex pris dans son ensemble ne tend pas à s'accélérer.

b) Supposons que la rotation initiale se fasse en masse (rotation terrestre par exemple). On a, au temps $t=0, \mathrm{~V}_{t}=\omega r$; et on trouve aisément la solution générale :

$$
V_{t}=\omega r e^{2 \mathrm{Ct}}
$$

Ainsi nous retrouvons un emballement de la rotalion, mais cette fois il est à loi exponentielle donc très explosif; et ceci n'est pas vrai seulement pour les anneaux individuels, mais aussi pour la rotation d'ensemble.

Le cas $(2 b)$ - que nous venons d'étudier diffère cependant essentiellement du cas $\left(\begin{array}{ll}1 & b\end{array}\right)$ que nous avons vu plus haut. Dans ce dernier cas, en effet, c'était un mouvement irrotationnel croissant linéairement avec le temps, qui se superposait au mouvement initial. Ainsi, en particulier, le rotationnel (d'axe vertical) du liquide restait constant en lout point. Dans le cas $(2 b)$, au contraire, c'est le mouvement de rotation « en masse » lui-mème qui s'emballe. Le rotationnel croît done exponentiellement dans toute la masse du liquide.

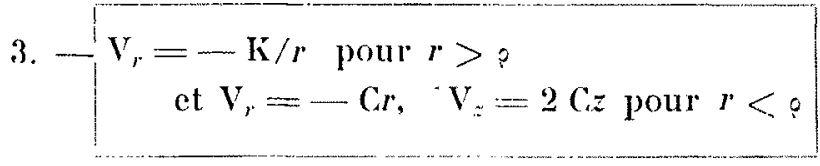

Essayons de faire la synthise des cats (1) ef (2) en admettant que l'ícoulement est assimilab'e à un écoulement plan autour d'un puits pour $r>$ ? et, pour $r<p$, à l'écoulement du lype (2)

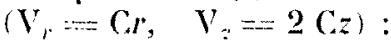

a) Si le vorlex est irrotationnel il se conserve identique dans les deux dommines. Ie monvement est permanent;

b) Si la rotation initiale se fail en masse, nous savons déjà ce qui se passe dans le domaine où $r$ est plus grand que o. A l'intéricur du cercle $r=e$, il faut distinguer deux cas. Le premier est celui des particules liquides qui, au temps $t=0$, se trouvaient dans le cercle $r=q$ : nous sarons que ces particules voient leur mouvement de rotation autour de l'axe s'accédérer exponenticllement. Le deuxieme cas est celui des particules entrćes dans le cercle de rayon apres le temps $t=0$ : pour celles-ci, l'équation $(1,2)$, qui peut s'écrire :

$$
\frac{1}{\mathrm{~V}_{t}} \frac{\mathrm{N} \mathrm{Y}_{i}}{\mathrm{D} t}=\mathrm{C}
$$

donne aisément le moyen de suivre leur évolution. Chaque anneau liquide voit, au cours de son évolution, sa vitesse tangentielle s'accrnitre en fonction du temps suivant la loi :

$$
\mathrm{V}_{t}(\text { anneau donné })=e^{\mathrm{C} t}
$$


L'évolution du mouvement est reproduite à titre d'exemple sur la planche I.

4. - Conclusion : La façon même dont l'équation (1) a été obtenue, en considérant individuellement l'histoire de chaque cylindre de liquide ayant $\mathrm{O} z$ pour axe, montre que, si l'on néglige la viscosité, c'est-à-dire toute réaction de ces cylindres l'un sur l'autre, on verra s'accentuer le mouvement de rotation de ceux d'entre eux qui ont toujours tourné, mais on n'en verra jamais aucun prendre de lui-mêne un mouvement de rotation s'il n'en avait pas initialement. Si les anneaux qui tournent se trouvent tous $\dot{a}$ distance finie, ils finissent tous par être « avalés » par le «puits » et laissent en définitive un écoulement dénué de toute rotation.

\section{B) VISCOSITÉ NON NÉGLIGEE}

1. - On constale aisément, et avec satisfaction, que les solutions étudiées en $(1 a),(1 b)$, $(2 a),(2 b)$ et $(3 a)$ sont valables même si l'on décide de tenir compte de la viscosité du fluide.

Ceci est vrai aussi, dans la solution $(3 b)$, pour les parties où la rotation se fait. en masse et pour celles où elle se fait suivant la loi du vortex, mais ce n'est plus vrai dans la zone de raccordement.

Il nous semble cependant très vraisemblable que la solution obtenue en $(3 b)$ représente d'une façon excellente l'écoulement qui résulterait de nos hypothèses complétées par la prise en compte de la viscosité, c'est-à-dire en définitive un écoulement assez voisin de ce qui doit arriver tans la réalité.

2. - Etude du cas général de l'écoulement plan autour d'un puits :

$$
\left(\mathrm{V}_{r}=-\frac{\mathrm{K}}{r}\right)
$$

L'équation (1) s'écrit dans ce cas :

$$
\begin{aligned}
\frac{\partial V_{t}}{\partial t}=\nu\left(\frac{\partial^{2} V_{t}}{\partial r^{2}}+\frac{1}{r}\right. & \left.\frac{\partial V_{t}}{\partial r}-\frac{1}{r^{2}}\right) \\
& +K\left(\frac{1}{r} \frac{\partial V_{t}}{\partial r}+\frac{1}{r^{2}} V_{t}\right)
\end{aligned}
$$

On peut vérifier que l'expression :

$$
\mathrm{V}_{t}=\mathrm{Ar}^{-n} J_{n-1}(\beta r) e^{-\beta^{2} p t}
$$

$\mathbf{J}_{n-1}$ étant la fonction de Besser d'ordre $n-1$;

$(n=(\mathrm{K} / 2 \nu) ; \beta$ : réel positif quelconque;

A : constante)

convient comme solution.
La solution générale résulte d'une combinaison linéaire de termes de cette forme.

On peut ainsi, grâce au théorème de FourierBEssse, former la solution correspondant à une distribution arbitraire des vitesses initiales (au temps $t=0$ ). Soit :

$$
\mathrm{V}_{t}=f(r) \quad \text { pour } t=0
$$

cette distribution initiale; on en tire :

$V_{t}=\int_{0}^{\infty} d \beta \int_{0}^{\infty} \beta \rho^{n+1} f(\rho) r^{-n} J_{n-1}(\beta \rho) J_{n-1}(\beta r) e^{-\beta^{n} r^{t}} d \rho$

On peut transformer l'intégrale en $\beta$ grâce à la formule de Weber. Il vient alors :

$$
\begin{array}{r}
\mathrm{V}_{t}=\frac{r^{-n}}{2 \nu t} e^{-r^{2} /(4 \nu t)} \int_{n}^{\infty} f(\rho) \rho^{n+1} e^{-\rho^{2} /(4 \nu t)} \mathrm{I}_{n-1} \\
\left(\frac{r}{2 \vee t} \rho\right) d \rho
\end{array}
$$

$I_{n-1}$ étant la fonction de Bessel modifiée d'ordre $\pi-1$.

Cette dernière solution donne donc la solution cherche par une quadrature. Elle permet d'ailleurs de tirer immédiatement une conclusion importante sur l'évolution du mouvement lorsque le temps tend vers l'infini.

En effet, si nous supposons que le mouvement de rotation initial $\mathrm{V}_{t}=f(r)$ est nul pour $r>\mathrm{R}$, $R$ étant une limite finie aussi grande que l'on veut, l'intégration peut se faire de 0 à $R$ au lieu de 0 à l'infini et par conséquent o reste borné sous le signe somme. On voit alors facilement que les termes intégrés tendent vers zéro dans tout le domaine fini d'intégration. $V_{t}$ a donc une limite nulle quand le temps tend vers l'infini.

En termes plus concrets, cela signifie que le mouvement de rotation ne peut se perpétuer que si il préexiste déjà jusqu'à une distance infinie. Si seule une zone finie autour de l'origine a initialement un mouvement de rotation, ce mouvement tend à disparaître (et finit par s'évanouir). L'évanouissement rapide (d'allure exponentielle) du mouvement de rotation a lieu lorsque toute la partie du liquide initialement en rotation a été «absorbée » par le puits.

\section{C) INFlufence DE LA SURFACE LibRE}

Dans tout ce qui précède, nous avons nẻgligé la perturbation due à la surface libre. Sans pouvoir donner une étude précise de cette perturbation, nous allons cependant faire quelques remarques sur deux cas particuliers :

$1^{\circ}$ Si les vitesses de l'écoulement ne sont pas trop grandes, il est probable que nos résultats 
conservent à peu près leur validité, la surface libre restant approximativement plane.

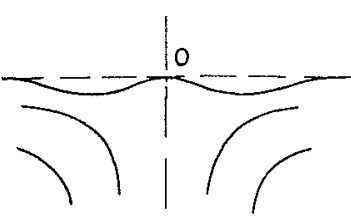

sons vorlex

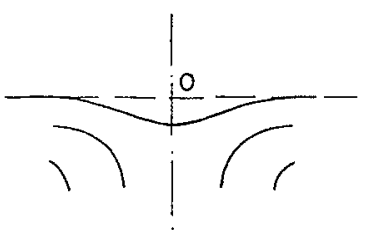

avec vorlex
Fig. 3

La figure 3 montre quelle pent être l'allure de la coupe méridienne d'un tel écoulement.

La présence d'une déformation de la surface dans la zone $O$, où précisément les vitesses sont faibles, n'a au fond que peu d'importance sur l'écoulement dans son ensemble;

$2^{\circ} \mathrm{Si}$ les vitesses de rotation sont très grandes au voisinage de l'axe, nous avons vu, dans les trois exemples étudiés, que la loi de répartition de la vitesse itait sensiblement celle du vor- tex. L'écuation de la méridienne de la surface libre est donc sensiblement (au voisinage de l'axe où les vitesses tangentielles $V_{t}=C / r$ sont prépondérantes) :

$$
z=-\frac{\mathrm{C}^{2}}{2 g r^{2},} \text { soit }: z r^{2}=\mathrm{C}^{\mathrm{te}}
$$

Or, cetle dernière équation est précisément celle des méridiennes des surfaces de courant de l'écoulement à trois dimensions que nous avons

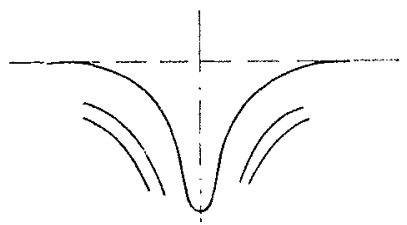

Fici. 4

étudié (fig. 4). Là encore la perturbalion sera donc peu importante.

\section{CONSULTATION PRELIMINAIRE POUR L'EXECUTION EVENTUELLE DES TRAVAUX DE GENIE CIVIL DU BARRAGE ET DE L'USINE DE SOUAPITI EN GUINÉ FRANÇAISE}

A l'initiative de la Société ENERGIE ELECTRIQUE DE GUINEE, un dossier d'appel d'offres a été préparé pour les travaux de génie civil de la chute de SOUAPITI sur le KONKOURE en Guinée Française. Ces travaux comporteraient, avec l'exécution des travaux préparatoires:

- D'une part, la construction d'une digue en latérite d'une hauteur de $120 \mathrm{~m}$ et d'environ 24 millions de $\mathrm{m}^{3}$ de remblais;

- D'autre part, les ouvrages annexes souterrains: évacuateurs de crues, galeries d'amenée et galerie de fuite, les deux galeries de dérivation provisoire étent équipées l'une en évacuateur de crues, l'autre en vidange;

- Enfin, le génie civil de l'usine souterraine (puissance installée : 4 groupes de $100.000 \mathrm{~kW}$ ).

Les délais d'exécution seraient de cinq à six ans à partir de la désignation de l'entrepreneur.

Les entreprises ou groupements d'entreprises qui seraient intéressés par les travaux projetés sont priés de se faire connaître à l'ELECTRICITE DE FRANCE, Ingénieur-Conseil el représentant à PARIS de l'ENERGIE ELECTRIQỤE DE: GUINEE, dans un délai de trois mois.

lls feront connaître les moyens en matériel dont ils disposeraient, les moyens qu'ils compteraient se procurer et, éventuellement, ceux qu'ils demanderaient au Maître de l'Guvre de mettre à leur disposilion.

ENERGIE ELECTRIQUE DE GUINEE se réserve de donner suite ou non aux candidatures qui seront présenlées. Il sera largement tenu compte dans l'appréciation de la capacité des Entreprises ou groupements à soumissionner, de l'importance et de la qualité des concours qu'ils seraient en mesure de s'assurer sous forme de Conseils, d'Association ou de locution de services auprès d'Entreprises ou d'Ingénieurs ayant une expérience effective de l'exécution de travaux de cette nature.

Les candidalures seront reçues par le Service des Etudes d'Outre-Mer de l'ElECTRICITE DE FRANCE, 77, avenue Raymond-Poincaré, PARIS (16") qui communiquera aux intéressés tous les renseignements et les dossiers nécessaires avec les caractéristiques essentielles des ouvrages.

Pour la visite du site des ouvrages, les Entreprises s'adresseront à l'ENERGIE ELECTRIQUE DE GUINEE, Boîte Postale 322 à CONAKRY, qui leur facilitera leurs déplacements.

La présente information ne préjuge en rien de la décision qui sera prise sur le principe même de la construction des ouvrages. 